\title{
Statistical limits of carrier density related low frequency theories in M.O.S. devices
}

\author{
A. A. Walma \\ Centre d'Etudes d'Electronique des Solides $(*)$, \\ Université des Sciences et Techniques du Languedoc, 34060 Montpellier Cedex, France \\ (Reçu le 6 octobre 1980, révisé le 18 décembre 1980, accepté le 18 décembre 1980)
}

\begin{abstract}
Résumé. - La densité de porteurs libres dans les théories relatives aux surfaces est considérée comme la projection d'un système à $n$ dimensions. Les variances de l'ensemble grand canonique sont utilisées pour résoudre le problème à plusieurs niveaux dans l'approximation de la diffusion. Il en découle une superposition de spectres qui évite de mettre en jeu une distribution en $1 / \tau$. Les origines cinétiques des constantes de temps sont discutées. Les conditions imposées conduisent à des contradictions dans les théories relatives aux densités de porteurs associées à des dispositifs M.O.S. réels. On montre que le spectre en $1 / f$ ainsi établi provient d'un processus stationnaire, Gaussien, non Markovien qui n'est pas en équilibre thermodynamique.
\end{abstract}

Abstract. - The free density in surface related theories is considered as a projection of a $n$-dimensional system. Grand canonical variances are used to solve the multi-level problem in the diffusion limit. A natural superposition occurs that avoids the $1 / \tau$ idea. The kinetic origins of the time constants are discussed. The imposed conditions lead to contradictions in carrier density related theories of a real M.O.S. device. The derived $1 / f$ spectrum is shown to arise from a stationary, Gaussian, non-Markovian process that is not in thermodynamic equilibrium.

\section{List of symbols.}

normalizing constant of $1 / \tau$ probability density function,

AA adiabatic assumption,

$\AA \quad$ angström,

C

$C(t)$ autocovariance,

$C_{n n}(t)$ covariance of level $n$,

$E$ level energy,

$E_{\mathrm{F}} \quad$ Fermi energy,

$e$ electronic charge,

$\mu \quad$ mobility,

$f$ frequency,

(*) L.A. au C.N.R.S. $f_{i} \quad$ occupation $i$ th level,

G-R generation-recombination,

$h \quad$ Planck's constant,

I identity matrix,

I total current in the sample,

$k \quad$ Boltzmann's constant,

$k_{i j} \quad$ element of matrix $\mathbf{M}_{i j}$,

$\lambda_{1,2}$ channel length of a M.O.S. device,

reciprocal values of $\tau_{1}$ and $\tau_{2}$,

M relaxation Matrix of a three variable Markov process,

$\mathbf{M}_{i j}$ relaxation Matrix of a three variable Markov process with $i$ an $j$ being the independently varying levels,

$m^{*} \quad$ effective mass,

$N$ total number of free carriers in the sample,

$N_{i} \quad$ total number of states of level $i$,

$N_{\text {ox }}$ total number of occupied states in the oxide at a certain energy,

$n_{0} \quad$ equilibrium electron density,

$n_{i} \quad$ fluctuating number of carriers at level $i$,

$n^{\prime} \quad$ number of free carriers if the Fermi level is at level one,

$n_{i}^{0} \quad$ deterministic equilibrium value of $n_{i}$,

OM Onsager-Machlup,

$p(\tau) \quad$ probability density function of $\tau$, 
$p_{0} \quad$ equilibrium hole density,

$R$ ohmic resistance,

$S_{I}(f)$ current spectral density,

$S_{N}(f)$ number spectral density,

$S_{n n}(f)$ number spectral density of level $n$,

$S_{n k}(f)$ cross spectral density of levels $n$ and $k$,

$T$ temperature in degrees Kelvin,

$\tau$ time constant single variable process,

$\tau_{1}, \tau_{2}$ lower and upper bound of $\tau$,

$\tau_{0} \quad$ smallest $\tau$,

$\tau_{k} \quad$ time constant in $k$-variable Markov process,

$\tau_{\mathrm{t}} \quad$ transit time,

$\tau_{\mathrm{po}} \quad$ Shockley lifetime of holes in extrinsic n-type material,

$\tau_{\text {no }} \quad$ Shockley lifetime of electrons in extrinsic ptype material,

$V_{\mathrm{D}} \quad$ drain voltage,

$W$ energy barrier between $\mathrm{Si}$ and $\mathrm{SiO}_{2}$,

$y$ transversal oxide distance,

$y_{1}, y_{2}$ lower and upper bound of $y$,

$\sigma_{i} \quad$ grand canonical variance of $i$ th level,

$\Delta \quad$ determinant of $\mathbf{M}_{n k}$.

1. Introduction. - The fast developments in SiTechnology of the last decade have greatly contributed to a better engineering and physical understanding of the $\mathrm{Si}_{-} \mathrm{SiO}_{2}$ system. Today's trend is towards submicron devices which implies that the physical system may become so small in the thermodynamic sense, that first order characterizations can become inaccurate. Parallel to this, the rapid progress in the amorphous field has resulted in a more special interest of the M.O.S. system. It appeared to be a strong candidate (at very low temperatures) for studying more fundamental phenomena experimentally with respect to the two dimensional $n$-body problem. These interpretations, together with to-day's refined technology, throws up the question whether previous theories still can be applied. In this paper we are interested in surface related $1 / f$ noise.

Low frequency noise is an almost universal phenomenon for which no generally accepted theory exists. In physical devices it is well known that the surface plays an important role. Numerous efforts have been undertaken in trying to find a theoretical explanation in M.O.S. devices with a varying degree of success. It is the only occasion, where some realistic predictions have been made.

The large majority of those $1 / f$ theories make a tacit use of a mathematical device that has been known for a long time [1, 2, 3] and a physical interpretation that was worked out in [4]. These ideas are the corner stones of the modified versions in [5-10] (see also for further refs.). It has been shown in [11] that this mathematical device is incompatible with the OnsagerMachlup theory in the single time constant approximation. In this paper it is investigated whether this single $\tau$ process is not a projection of a multi-dimen- sional process and whether it can provide us with a $1 / f$ type of spectrum. The spectral properties of a simplified $n$-dimensional problem are solved exactly. The imposed approximations are those that are in current use in surface related theories.

It is not our intention however to present some new $1 / f$ theory. Fundamental questions such as stationarity, Gaussienity, Markovian or not, Equilibrium, surface or bulk effect, are only discussed within the context of the socalled McWorther model. It is simply dealt with the question why a straightforward model, such as a superposition of relaxation spectra, can give a realistic prediction of a complex phenomenon like $1 / f$ noise in such a complex system as the $\mathrm{Si}_{-} \mathrm{SiO}_{2}$ interface. In other words, the statistical limits of carrier density fluctuations as an explanation of $1 / f$ noise in physical devices are discussed.

In the original contribution, two ways of obtaining a $1 / f$ spectrum have been suggested as a result of field effect experiments on Ge. The first assumes that the time constant of the slow states varies over the surface with a $1 / \tau$ distribution and the second takes the surface barrier into account and includes a modulation. Although recent advances in surface physics suggests a somewhat different interpretation, these ideas have been used in numerous treatments of $1 / f$ noise and led to realistic predictions on various occasions. In an attempt to find out why this is so, we shall be concerned with the following questions :

(i) what is the nature of the socalled shot-noise spectra?

(ii) what are the origins of the $1 / \tau$ distribution ?

(iii) what does the averaging procedure really represent?

The information about these basic elements and the choice of the fluctuating quantity are not very exhaustive neither in the original contribution nor in the following ones. The analysis becomes even more difficult if the bond switching idea is accepted near the surface. The trap density then becomes a dynamical quantity $[15,16]$. Points (i) an (ii) will therefore be considered from the usual SHR point of view using G-R theory. The results in the multi dimensional case will be more explicit than those from [13] and [14] by using grand-canonical variances and by avoiding the Boltzmann-Approximation (BA).

In the original contribution and subsequent treatments the averaging procedure is accepted without criticism. Decomposing a complex problem into more simple basic processes may not work statistically, however. Besides, the majority of the treatments only consider the decaying properties of these basic processes. This is a little unexpected since the stationary variance is closely related to the relaxation mechanism. It will be shown that the socalled averaging only can be carried out under certain conditions. They are not always satisfied in surface related theories. It is suggested that the right order of magnitude of 
any prediction is mainly due to the inherent use of a strong statistical and physical argument and not so much to the proposed theory.

2. The model. - The modifications of the model from [4] are not consistent with respect to the choice of the fluctuating variable or the $1 / \tau$ and modulation idea. We therefore reproduce an example from literature [9] that is characteristic to our opinion and which contains the necessary elements for our discussion. It was able to explain Hooge's formula in a remarkable straightforward way on the basis of carrier density fluctuations (which does not mean that the origins of this formula are now clarified of course). This suggests an intuitively correct application of certain strong physical and statistical arguments that lead to a realistic result since the method as such contains some contradictions.

(i) Let the number $N$ of free carriers in the sample fluctuate by $N$ due to communication with the oxide traps.

(ii) Let this fluctuation be governed by a time constant $\tau$ such that the autocovariance can be written as

$$
C(t)=\overline{\Delta N}^{2} \mathrm{e}^{-t / \tau}
$$

(iii) The current $I$ is proportional to $N$ and since a single carrier causes a current of $e / \tau_{\mathrm{t}}$ with $\tau_{\mathrm{t}}$ being the transit time, the current spectral density can be written as

$$
S_{I}(f)=\frac{I^{2}}{\bar{N}^{2}} S_{N}(f)
$$

(iv) Take into account a non-poissonian behaviour for the variance and write

$$
\overline{\Delta N}^{2}=\beta(\bar{N}) \bar{N}
$$

where $\beta(\bar{N})$ stands for the surface efficiency (see [6] and [9] for instance).

(v) Take for the time constant the following dependency

$$
\tau=\tau_{0} \mathrm{e}^{\alpha y}
$$

where $y$ represents in the above case the trap distance in the oxide.

(vi) Assume a uniform trap distribution between $y_{1}$ and $y_{2}$ yielding the following distribution for the time constants

$$
g(\tau) \mathrm{d} \tau=\frac{\mathrm{d} \tau / \tau}{\ln \tau_{2} / \tau_{1}}, \quad \tau_{1}<\tau<\tau_{2}
$$

with $\tau_{1}=\tau_{0} \mathrm{e}^{\alpha y_{1}}$ and $\tau_{2}=\tau_{0} \mathrm{e}^{\alpha y_{2}}$. (vii) Apply Wiener-Khintchine to the autocovariance (1) and average over $\tau$ :

$$
S_{I}(f)=4 \frac{\beta(\bar{N}) I^{2}}{\bar{N}} \int_{0}^{\infty} \frac{\tau g(\tau) \mathrm{d} \tau}{\omega^{2} \tau^{2}+1} \cong \frac{I^{2}}{\bar{N}} \alpha_{\mathrm{H}} \frac{1}{f}
$$

which is Hooge's formula with

$$
\alpha_{\mathrm{H}}=\frac{\beta(\bar{N})}{\ln \tau_{2} / \tau_{1}}
$$

We shall not discuss its validity since we are interested in the statistical limits of this procedure.

Other treatments that are different but still modifications from the ideas in [1-4] contain the same ingredients that are of interest to us in a more general sense. These problems can now be stated a bit more explicitly than was done in the introduction :

(i) What is the nature of the single time constant approximation.

(ii) The basic process given by (1) constitutes an Ornstein-Uhlenbeck process [11]. Are its conditions realistic for surface related $1 / f$ noise.

(iii) What is the nature of $\beta$ in (3).

(iv) Does the time constant given by (4) take the microscopic rates adequately into account.

(v) What does the averaging procedure from equation (6) really represent.

3. The single time constant approximation. - It has been customary in semiconductor statistics to represent the time dependent properties of the process under study by a single time constant. Examples are the generation-recombination (G-R) noise treatments in electronic devices $[13,14]$ and the interpretation of photo transients, electric responses, photo conductivity experiments and surface related methods in general, be it in crystalline or amorphous materials [22]. This is not sufficient in general for $1 / f$ explanations. Complex single variable processes have the tendency in the thermodynamic limit to converge to an Ornstein-Uhlenbeck $(\mathrm{O}-\mathrm{U})$ process and do therefore not yield $1 / f$ noise. For more than one variable the complexity of the calculations goes rapidly up and a usefull comparison with experimental results becomes difficult. One therefore looks for an overall relaxation time that takes the essential features of the phenomenon under study adequately into account. In order to avoid the O-U process, the only way to produce a $1 / f$-type of spectrum is then a non-linear or nonMarkovian process. Theories in the past all use the Markovian assumption and consider systems that can be linearized. This excludes the single time approximation as a candidate for $1 / f$ noise. One therefore looks for a usefull superposition. A complication arises however with respect to these classical treatments when applied in disordered structures. Here, the propagation appears to be non-Gaussian and non-Markovian [23]. 
Previous work on the macroscopic treatment of noise has been summarized in [14]. Even today (at least for electronic devices) the same ideas are still in use and hold particularly for surface related $1 / f$ theories. They can be summarized as follows [14] :

(i) The system is Markovian, stationary and linear (or can be linearized).

(ii) The system obeys time reversibility.

(iii) Fluctuations are small and from an equilibrium state.

(iv) The variables have a Gaussian distribution (or can accurately be approximated by it).

It was shown in [11] that the usual superposition of relaxation spectra yields a non-Markovian process. The $1 / \tau$ idea is therefore of importance. Most physical systems are Markovian however. It is then natural to ask whether the interface is non-Markovian or whether we have overlooked something in the analysis and whether there exists a way to explain a $1 / f$ type of spectrum from a Markovian system. That the latter possibility is not unrealistic can be seen as follows. Each system has a small number of integrals of motion. These variables vary at a rate that can be observed macroscopically. In our case such a variable is the carrier density. The microscopic relevant variables (transition rates for instance) vary so fast that the physical system quickly forgets the initial values of the nonrelevant variables. The problem is now to choose a sufficiently complete set of variables sothat any future development is perfectly described by the present values. This has been introduced as a principle of statistical mechanics of time dependent phenomena in [24]. It was argued that random processes associated with a complete set of variables are stationary Markov processes. Since the $1 / \tau$ idea yields a non-Markovian process [11], we therefore have to ask whether the carrier density is not a projection of different processes or whether the choice of this macroscopic variable in the single time constant approximation does not represent a complete set in the above sense. In the latter case there will be no $1 / f$ spectrum. For the averaging or projection however, the nature of the time constant is of vital interest. Their origins are discussed in the following sections.

4. The single state process. - In the Markov sense the time dependent properties of any system can be characterized by one or more time constants that are functions of the microscopic properties of the system, usually given by rate constants. The nature of the time constant(s) determine(s) the process and vice versa. The time constant given by equation (4) in the model is either a contraction of a more complicated expression or simply the time constant of a single state process. The latter possibility immediately imposes certain conditions upon the $1 / f$ explanation that need not to be satisfied in practice. Before discussing this process (and subsequent models) the following notation is introduced that will be used throughout this paper :

$n_{i}=$ Fluctuating number at the energy level $i$, where $i=1$ represents the lowest energy.

$N_{i}=$ Total number of states at energy $i$.

$a_{i}=$ Probability per unit of time that a transition takes place from level $i$ to level $i+1$ (Release).

$b_{i}=$ Probability per unit of time that a transition takes place from level $i+1$ to level $i$ (Capture).

In the single level case the increase per unit of time in the population is a constant. The decrease depends on the size of the population. The rate equation becomes :

$$
\frac{\mathrm{d} n_{1}}{\mathrm{~d} t}=b_{1}-a_{1} n_{1} .
$$

The time constant is given by $a_{1}^{-1}$. If this release (or capture in oxide for instance) has an activation energy or is exponentially depending on some distance, the choice of $\tau$ in equation (4) can be interpreted physically. The model as such is now in error : (i) The variance (G-R theorem) is $b_{1} / a_{1}$ and thus Poisson. This implies a surface state efficiency equal to one. (ii) The variance also contains the time constant and should be included in the averaging. No $1 / f$ spectrum arises but a $1 / f^{2}$ spectrum instead. (iii) It is physically difficult to explain how the increase of the free carriers $n_{1}$ can be a constant. The single time constant as is given by equation (4) needs a different process which inevitably complicates the analysis and the physical interpretation.

A more subtle difficulty arises with respect to the nature of the rate constants. It is always taken for granted that they constitute uncorrelated processes. This is not so clear at the interface. Polaron effects, thermal activated transport, paired states and VAP's in general modify the usual trapping ideas. An example for the single state process, with $a_{1}$ being nonadditive, showed a drastic change in the timedependency [37]. Surface related theories use the classical ideas from section three however, which includes the nonadditivity of the rate constants.

5. The two-state process. - This process is closely related to Fermi-Dirac statistics. Its variance resembles the grand canonical one. It has been used on various occasions without checking the criteria for its validity. In physical devices it can be found under various headings. (i) Random switch model. (ii) Random Telegraph signal [25]. (iii) Two parameter model [4]. (iv) Machlup process. In stochastic literature the process is well-known and can be considered as a two-state Markov process in continuous time [27]. It is one of the simplest stationary processes and has a complete solution, since the partial differential equation of the probability transform is linear. 
Suppose that there are $N_{1}$ electronic states obeying the Pauli exclusion principle. The increase of the occupied states is then proportional to a certain rate $b_{1}$ and the open places, whereas the decrease is proportional to the rate $a_{1}$ and the number of occupied states. The following rate equation arises :

$$
\frac{\mathrm{d} n_{1}}{\mathrm{~d} t}=b_{1}\left(N_{1}-n_{1}\right)-a_{1} n_{1} .
$$

The stationary variance can be written as (G-R theorem) :

$$
\overline{\Delta n_{1}^{2}}=\frac{a_{1} b_{1}}{\left(a_{1}+b_{1}\right)^{2}} N_{1}=f_{1}\left(1-f_{1}\right) N_{1}, \quad f_{1}=\frac{a_{1}}{a_{1}+b_{1}}
$$

and the time constant :

$$
\tau=\frac{1}{a_{1}+b_{1}} .
$$

As can be seen from (11), it is now more difficult to account for the single time constant such as is given by equation (4). The rates, and therefore $\tau$, depend on the choice of the fluctuating variable (trapped or free carriers for instance). Also for this case, the variance is a function of the time constant. The role of the steps (iv), (v) and (vii) in section two therefore depends on the choice of the physical model. Generalising the McWorther ideas such as is done in the quoted example is then making a reference to some unknown physical model or accepting the implicit use of certain strong physical and statistical arguments.

Surface theories make extensive use of (9), (10) and (11). It is then usually referred to [14] and [25]. The stationary statistics are taken from the grand canonical ensemble. Basically, it is then referred to the Einstein interpretation of the Boltzmann principle. The definition of the entropy function is extended to a class of non-equilibrium states and used to establish a probability density function. It has been shown in [29] however that this need not to be valid in a strongly interacting system. Problems arise with large trap densities, kinetics at the Fermi level, Polaron effects, interactions at the same energy etc. The thermodynamic result used in $[5,7,8,10]$ a.o. therefore imposes upon their theory : (i) A Heatbath assumption (ii) The large system approximation (iii) Small relative fluctuations (iv) Ergodicity (v) Independent particle system.

6. Two level statistics. - Quite popular amongst device physicists is the SHR statistics [33]. For additive microscopic processes it takes the time dependent properties adequately into account. The fluctuating heatbath is in our case the number of free carriers and the macroscopic rate equations become non-linear. A rigorous statistical treatment within the context of a stationary Markov process with local changes has not been carried out yet. We suppose however (this is usually so in surface related theories) that a diffusion approximation in the stochastic sense, such as given by the G-R theorem or the simple Langevin (white source) approach, is accurate enough to take the nonlinear character into account. An example that this is often so can be found in [29]. It is one of those rare occasions where the diffusion approximation is compared with the exact equilibrium statistics. Almost exact equilibrium statistics does not imply the same for the time dependency however. For weak restoring properties, even the equilibrium results have to be handled with care [31]. This is important for $1 / f$ theories since the relaxation toward equilibrium is now more complicated than the G-R theorem suggests. In order words it remains to be seen whether SHR kinetics always relaxes back in a fashion that can be described by a single time constant. In this paper the restoring is supposed to be strong enough however.

Consider the kinetics for a single carrier [33] :

Rate of capture $=\left(1-f_{t}\right) N_{t}(E) c_{n} f(E) N(E) \mathrm{d} E$

Rate of release $=f_{t} N_{t}(E) e_{n}[1-f(E)] N(E) \mathrm{d} E$

where $E$ stands for the level energy and $f_{t}$ the trap occupancy with $N_{t}(E)$ being its total number. For bulk traps the rate constant $c_{n}$ depends on the thermal velocity and the capture crossection while $e_{n}$ is a function of the phonon frequency and the level energy. For traps in the oxide, other physical parameters may determine these constants. Keeping things as simple as possible, we ignore the energy dependence and consider the following rate equations on the basis of (12) and (13) :

$$
\begin{aligned}
\frac{\mathrm{d} n_{1}}{\mathrm{~d} t} & =a_{1} n_{2}\left(N_{1}-n_{1}\right)-b_{1} n_{1}\left(N_{2}-n_{2}\right) \\
\frac{\mathrm{d} n_{2}}{\mathrm{~d} t} & =b_{1} n_{1}\left(N_{2}-n_{2}\right)-a_{1} n_{2}\left(N_{1}-n_{1}\right) \\
N & =n_{1}+n_{2} .
\end{aligned}
$$

In our case the subscript two refers to the conduction band and one to the traplevel. Applying the G-R theorem $[13,14]$ :

$$
\overline{\Delta n_{1}^{2}}=\overline{\Delta n_{2}^{2}}=\frac{n_{1}^{0} n_{2}^{0}\left(N_{1}-n_{1}^{0}\right)\left(N_{2}-n_{2}^{0}\right)}{\left(N N_{1}-n_{1}^{02}\right)\left(N_{2}-n_{2}^{0}\right)+n_{1}^{0} n_{2}^{0}\left(N_{1}-n_{1}^{0}\right)}
$$

where the superscript zero refers to the deterministic equilibrium value. For the time constant we get :

$$
\tau=\frac{n_{1}^{0}\left(N_{2}-n_{2}^{0}\right)}{a_{1}\left(N N_{1}-n_{1}^{0^{2}}\right)\left(N_{2}-n_{2}^{0}\right)+a_{1}\left(N_{1}-n_{1}^{0}\right)\left(N-n_{1}^{0}\right) n_{1}^{0}} .
$$

These equations are difficult to work with and can hardly be used for a physical interpretation. Fortuna- 
tely they can be simplified considerably in terms of the grand canonical variances $f_{1}\left(1-f_{1}\right) N_{1}$ and

$f_{2}\left(1-f_{2}\right) N_{2}$.

Denoting these by $\sigma_{1}$ and $\sigma_{2}$ respectively :

$\overline{\Delta n_{1}^{2}}=\overline{\Delta n_{2}^{2}}=\frac{\sigma_{1} \sigma_{2}}{\sigma_{1}+\sigma_{2}}, \quad \tau=\frac{f_{1}\left(1-f_{2}\right)}{a_{1}\left(\sigma_{1}+\sigma_{2}\right)}$

where we have used the equilibrium condition :

$$
\frac{a_{1}}{b_{1}}=\frac{f_{1}\left(1-f_{2}\right)}{f_{2}\left(1-f_{1}\right)}=\mathrm{e}^{\left(E_{2}-E_{1}\right) / k T} .
$$

If the system, given by (14) and (15), converges in its parameters to a system that is describable by a FokkerPlanck equation, then in the thermodynamic limit the results (18) are exact. In our simple case these conditions are satisfied and the nonlinear equations can be linearized. This may not be so at the interface (correlated capture and release for instance).

Frequently one encounters in noise literature the following modification of (our notation) (14) :

$$
\frac{\mathrm{d} n_{1}}{\mathrm{~d} t}=a_{1} n_{2}\left(N_{1}-n_{1}\right)-a_{1} n_{1} n^{\prime}
$$

It implies the Boltzmann-Approximation however. It was shown in [34] that for a set of noninteracting electrons in the nondegenerate case the mean number of electrons is given by (our notation) :

$$
n_{2} \cong N_{2} \mathrm{e}^{\left(E_{F}-E_{2}\right) / k T}
$$

with $E_{\mathrm{F}}$ the Fermi energy and $E_{2}$ the one of the conduction band. Placing the Fermi level at the height of the traplevel, a kinetic invariant was found in [33] and denoted by (our notation) :

$$
n^{\prime}=N_{2} \mathrm{e}^{\left(E_{1}-E_{2}\right) / k T} .
$$

From the equilibrium condition (19) it can be seen however that the use of quasi levels implies the Boltzmann-Approximation (BA) :

$$
\begin{aligned}
& \frac{a_{1}}{b_{1}}=\mathrm{e}^{\left(E_{2}-E_{1}\right) / k T} \Rightarrow n^{\prime}=\left(N_{2}-n_{2}\right) \mathrm{e}^{\left(E_{1}-E_{2}\right) / k T} \cong \\
& \cong N_{2} \mathrm{e}^{\left(E_{1}-E_{2}\right) / k T}
\end{aligned}
$$

At an interface or in a disordered structure, transport depends on the kinetics near the Fermi level so that the BA may not hold. This also counts for surface related $1 / f$ theories since the interactions with the oxide often take place at the same energy level.

Relaxing the heatbath assumption provides us with relatively simple expressions. As for the time constant more details about the physical process are now needed in order to obtain the $1 / \tau$ structure of equation (4). This can be difficult in a practical case since the real nature of the socalled slow states is not known yet and about the interaction kinetics confusion exists [10]. Restoring the heatbath assumption means in (18) that $\sigma_{2} \gg \sigma_{1}$ which gives us $\sigma_{1}$ back again (grand canonical variance). The time constant becomes :

$$
\begin{aligned}
\tau \cong \frac{f_{1}\left(1-f_{2}\right)}{a_{1} \sigma_{2}}=\frac{f_{1}\left(1-f_{2}\right)}{a_{1} f_{2}\left(1-f_{2}\right) N_{2}}= & \frac{f_{1}}{a_{1} f_{2} N_{2}} \cong \\
& \cong \frac{1}{b_{1} N_{2}}
\end{aligned}
$$

where we have used (19) plus the BA for the last equality. That this is a correct result can also be seen from (14). Using the BA :

$$
\frac{\mathrm{d} n_{1}}{\mathrm{~d} t} \cong a_{1} n_{2} N_{1}-b_{1} n_{1} N_{2}
$$

With $n_{2}$ acting as a heatbath (no fluctuations) the time constant is then determined by $b_{1} N_{2}$ as in (24).

More complete surface theories apply the full SHR statistics. This means the introduction of an extra variable. In the single time constant approximation the process has then become non-Markovian. Two time-constants are involved sothat for the Markovian case at least a two-variable process is needed. This also means that those theories using the SHR time constant in the single variable case are incompatible with the Onsager-Machlup theory for irreversible processes.

Those theories, using the grand canonical ensemble, also consider a two level problem. Level one is the trapping level for instance and level two the heat bath. This means in equation (18) that $\sigma_{2} \gg \sigma_{1}$ and for equation (14) that it can be written as :

$$
\frac{\mathrm{d} n_{1}}{\mathrm{~d} t} \cong a_{1} n_{2}\left(N_{1}-n_{1}\right)-b_{1} N_{2} n_{1}
$$

where $n_{2}$ is taken constant and the BA is applied. The variance is now of the grand canonical type with :

$$
f_{1}=\frac{a_{1} n_{2}}{a_{1} n_{2}+b_{1} N_{2}}=\left[1+\mathrm{e}^{\left(E_{1}-E_{\mathbf{F}}\right) / k T}\right]^{-1} .
$$

This shows once more that the use of the grand canonical variance implies a Heatbath assumption and the Boltzmann approximation for it. For a realistic physical interpretation of the required time constant given by equation (4) the two-level time dependency is a little complex. If the grand canonical variance is used, then the time constant is unfortunately the shortest one and given by $\tau=\left(b_{1} N_{2}\right)^{-1}$, which is equivalent to the Shockley life time for electrons in p-type material [33]. For deep traps the binding energies are probably too small to give appreciable long time constants. If $b_{1}$ is used with an exponential dependency with respect to the distance in the oxide, then the already mentioned conditions should be invoked. 
7. The three level problem. - The mathematical aspects of noise in the diffusion approximation for the multi level case have been treated extensively in [13] and [14]. Though the statistical nature of electronic processes in solids is basically of a non-linear character (Pauli exclusion principle), these treatments consider sufficiently sharply peaked transition probabilities sothat it can be linearized. The actual process is therefore assumed to be describable by a multidimensional O-U process [31]. In disordered structures this may not work since the propagation has a nonGaussian and non-Markovian character [23]. In [15, 16] it is suggested that an amorphous transition region exists at the $\mathrm{Si}_{-} \mathrm{SiO}_{2}$ interface in order to meet some discrepancies that arise with the physical interpretation of experimental evidence in surface related $1 / f$ noise theories. If this is correct than the diffusion approximation for a statistical treatment (G-R theorem, Langevin with a white source, Burgess' method etc.) may become inaccurate. We hope to come back upon this elsewhere. For a discussion of the existing theories, the notions from [13] and [14] are used.

Introducing a third level (SHR statistics) complicates the analysis considerably. The complete expressions from [13, 14] and [35] can hardly be used for a physical interpretation. Usually the noise is then calculated numerically in order to be able to derive the desired $1 / f$ dependency. The analytical treatment can be simplified however if one uses consistently the grand canonical variances for the respective levels. A more complete discussion will appear elsewhere. In this paper the relevant results for our case will be presented without comment. On the other hand, they reduce to the expressions that can be found in [13] and [14]. They will be used within the context of previous $1 / f$ theories that have applied the SHR idea for a more involved treatment of the time constant. Both [13] and [14] (also the thermodynamic method from [29]) make a free use of the BA. This may become inaccurate at the interface (it almost certainly is in amorphous materials where part of the kinetics takes place near the Fermi level).

In the one-dimensional case an $\mathrm{O}-\mathrm{U}$ process has an autocorrelation of the type [27,32] :

$$
C(t)=\overline{\Delta n^{2}} \mathrm{e}^{-\beta t} .
$$

Applying the Wiener-Khinchine theorem and going over to the multidimensional case :

$$
S(f)=4 \operatorname{Re}(\mathbf{M}+j \omega \mathbf{I})^{-1} \mathbf{C}
$$

where $\mathbf{M}$ represents the relaxation matrix [13, 14, 41] and $\mathbf{C}$ the correlation matrix (I is the identity matrix). For the relaxation matrix the following rate equations are studied :

$$
\begin{aligned}
& \frac{\mathrm{d} n_{1}}{\mathrm{~d} t}=a_{1} n_{2}\left(N_{1}-n_{1}\right)-b_{1} n_{1}\left(N_{2}-n_{2}\right) \\
& \frac{\mathrm{d} n_{2}}{\mathrm{~d} t}=a_{2} n_{3}\left(N_{2}-n_{2}\right)+b_{1} n_{1}\left(N_{2}-n_{2}\right)- \\
& -b_{2} n_{2}\left(N_{3}-n_{3}\right)-a_{1} n_{2}\left(N_{1}-n_{1}\right) \\
& \frac{\mathrm{d} n_{3}}{\mathrm{~d} t}=b_{2} n_{2}\left(N_{3}-n_{3}\right)-a_{2} n_{3}\left(N_{2}-n_{2}\right) \\
& N=n_{1}+n_{2}+n_{3} \text {. }
\end{aligned}
$$

From these equations it follows that it is dealt with a closed but non-cycling system without using the BA. The rate constants are assumed to originate from additive processes. In equilibrium it follows that :

$$
\frac{a_{1}}{b_{1}}=\frac{f_{1}\left(1-f_{2}\right)}{f_{2}\left(1-f_{1}\right)}, \quad \frac{a_{2}}{b_{2}}=\frac{f_{2}\left(1-f_{3}\right)}{f_{3}\left(1-f_{2}\right)} .
$$

Linearizing (30) and (32) by using the constraint (33), the relaxation matrix can be written as :

$$
\mathbf{M}_{13}=\left[\begin{array}{cc}
\frac{a_{1} \sigma_{1}}{f_{1}\left(1-f_{2}\right)}+\frac{a_{1} \sigma_{2}}{f_{1}\left(1-f_{2}\right)}, & \frac{a_{1} \sigma_{1}}{f_{1}\left(1-f_{2}\right)} \\
\frac{-a_{2} \sigma_{3}}{f_{2}\left(1-f_{3}\right)}, & \frac{a_{2} \sigma_{3}}{f_{2}\left(1-f_{3}\right)}+\frac{a_{2} \sigma_{2}}{f_{2}\left(1-f_{3}\right)}
\end{array}\right] .
$$

Abreviating this by :

$$
\mathbf{M}_{13}=\left[\begin{array}{ll}
k_{11}, & k_{12} \\
k_{21}, & k_{22}
\end{array}\right]
$$

the relevant spectral densities follow from (29) :

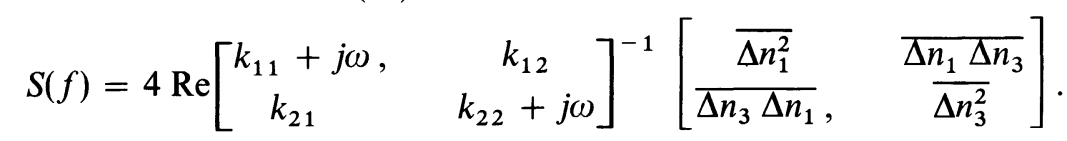

In these expressions $f_{1}, f_{2}$ and $f_{3}$ represent the respective occupancies and $\sigma_{1}, \sigma_{2}, \sigma_{3}$ the grand canonical variances.
For the stationary variance one could have used $1 / 2 \mathbf{M}^{-1} \mathbf{B}$ [13] [14] with $\mathbf{B}$ the matrix for the infinitesimal variances. It will be more convenient however 
to use the non-cycling idea of the two-level case where the levels are considered to be independent. For obtaining the variance of level one in the three variable case, the levels two and three are taken together and by using the two-level result equation (18) :

$$
\overline{\Delta n_{1}^{2}}=\frac{\sigma_{1}\left(\sigma_{2}+\sigma_{3}\right)}{\sigma_{1}+\sigma_{2}+\sigma_{3}} \text {. }
$$

It will be shown elsewhere in a more detailed discussion that this is also correct in the cycling case, provided that the socalled Wegscheider relations hold. This also counts for the non-cycling $n$-dimensional problem. Similarly :

$$
\overline{\Delta n_{3}^{2}}=\frac{\sigma_{3}\left(\sigma_{1}+\sigma_{2}\right)}{\sigma_{1}+\sigma_{2}+\sigma_{3}}
$$

and for the covariance, using the particle constraint :

$$
\overline{\Delta n_{1} \Delta n_{3}}=\frac{-\sigma_{1} \sigma_{3}}{\sigma_{1}+\sigma_{2}+\sigma_{3}} \text {. }
$$

For the (cros) spectral densities of levels one and two (or two and three), the relaxation matrix will be different. Here we are interested in the free carriers (level three). The spectral density becomes (see eq. (37)) :

$S_{33}(f)=4 \operatorname{Re} \frac{\left(k_{11}+j \omega\right) \overline{\Delta n_{3}^{2}}-k_{21} \overline{\Delta n_{1} \Delta n_{3}}}{\left(k_{11}+j \omega\right)\left(k_{22}+j \omega\right)-k_{12} k_{21}}$.

Applying a partial fraction expansion and using (35), (39) and (40) :

$$
\begin{aligned}
S_{33}(f)=4 \overline{\Delta n_{3}^{2}} \operatorname{Re}\left[\frac{A+\lambda_{1}}{\lambda_{1}-\lambda_{2}} \frac{1}{\left(\lambda-\lambda_{1}\right)}+\right. \\
\left.\quad+\frac{A+\lambda_{2}}{\lambda_{2}-\lambda_{1}} \frac{1}{\left(\lambda-\lambda_{2}\right)}\right]
\end{aligned}
$$

where $\lambda_{1}$ and $\lambda_{2}$ are the solutions of :

$$
\lambda^{2}-\left(k_{11}+k_{22}\right) \lambda+\left\|\mathbf{M}_{13}\right\|=0
$$

and

$$
A=\frac{\left(k_{11}+k_{21}\right) \sigma_{1} \sigma_{3}+k_{11} \sigma_{2} \sigma_{3}}{\sigma_{3}\left(\sigma_{1}+\sigma_{2}\right)}, \quad \lambda=j \omega .
$$

Taking the real part of (42) :

$$
\begin{aligned}
S_{33}(f)=4 \overline{\Delta n_{3}^{2}} & {\left[\frac{A+\lambda_{1}}{\lambda_{2}-\lambda_{1}} \frac{\lambda_{1}}{\omega^{2}+\lambda_{1}^{2}}+\right.} \\
& \left.+\frac{A+\lambda_{2}}{\lambda_{1}-\lambda_{2}} \frac{\lambda_{2}}{\omega^{2}+\lambda_{2}^{2}}\right] .
\end{aligned}
$$

The time constants of the three level problem follow from (43).

As can be seen, the complexity of the analysis goes rapidly up with the number of levels. Particularly with respect to the involved time constants, it is difficult to see how one arrives at a $1 / \tau$ distribution if all effects are taken into account. The macroscopic time dependency is a rather complicated function of the relevant microscopic processes sothat it will be difficult to concentrate upon a possible distribution of physical rates that are thought to be dominating. Surface related theories in general are therefore looking for some overall behaviour, and use the general ideas of the model from section two. Analytical difficulties are then masking physical detail.

Since certain theories use the SHR time constant for their averaging, it will be interesting to have a closer look at the solutions of (43). The roots satisfy :

$$
\left.\begin{array}{rl}
\lambda_{1}+\lambda_{2} & =k_{11}+k_{22} \\
\lambda_{1} \lambda_{2} & =k_{11} k_{22}-k_{12} k_{21}
\end{array}\right\}
$$

They can be written as [13] :

$\lambda_{1,2}=\frac{1}{2}\left(k_{11}+k_{22}\right)\left[1 \pm\left[1-\frac{4 \Delta}{\left(k_{11}+k_{22}\right)^{2}}\right]^{1 / 2}\right]$

where $\Delta=k_{11} k_{22}-k_{21} k_{12}=\left\|\mathbf{M}_{13}\right\|$. Consider now :

$\frac{4 \Delta}{\left(k_{11}+k_{22}\right)^{2}}=\frac{4 \lambda_{1} \lambda_{2}}{\left(\lambda_{1}+\lambda_{2}\right)^{2}}=\frac{4 \lambda_{1}}{\lambda_{1}+\lambda_{2}}\left[1-\frac{\lambda_{1}}{\lambda_{1}+\lambda_{2}}\right]$

where we have used (46). Writing for (47) :

$$
\lambda_{1,2}=\frac{1}{2}\left(\lambda_{1}+\lambda_{2}\right)\left[1 \pm(1-\varepsilon)^{1 / 2}\right]
$$

it can be seen from (48) that for $\lambda_{2}=3 \lambda_{1}$ the root expansion for (47) becomes already sufficiently accurate. This yields for the time constants :

$\tau_{1}=\frac{1}{k_{11}+k_{22}}, \quad \tau_{2}=\frac{k_{11}+k_{22}}{k_{11} k_{22}+k_{12} k_{21}}$.

Together with (35) :

$$
\begin{aligned}
\tau_{1} & =\frac{f_{1}\left(1-f_{2}\right) f_{2}\left(1-f_{3}\right)}{a_{1}\left(\sigma_{1}+\sigma_{2}\right) f_{2}\left(1-f_{3}\right)+a_{2}\left(\sigma_{2}+\sigma_{3}\right) f_{1}\left(1-f_{2}\right)} \\
\tau_{2} & =\frac{a_{1}\left(\sigma_{1}+\sigma_{2}\right) f_{2}\left(1-f_{3}\right)+a_{2}\left(\sigma_{2}+\sigma_{3}\right) f_{1}\left(1-f_{2}\right)}{a_{1} a_{2} \sigma_{2}\left(\sigma_{1}+\sigma_{2}+\sigma_{3}\right)}
\end{aligned}
$$


Modifications of these formulas can be found frequently in literature, either for calculating the SHR time constant or by using them for some averaging procedure. Always including the Boltzmann approximation, this statistics still is the most important tool for surface related theories. The equations show however how difficult it is to account statistically and physically for a $1 / \tau$ distribution. $1 / f$ theories therefore accept certain intrinsic simplifications that make a usefull physical interpretation doubtfull for more involved systems. They necessarily imply an adiabatic assumption for example in order to be able to linearize. Using this property $\left(\sigma_{3} \gg \sigma_{2}, \sigma_{1}\right)$ for the time constants :

$$
\begin{aligned}
& \tau_{1} \cong \frac{f_{1} f_{2}\left(1-f_{2}\right)\left(1-f_{3}\right)}{a_{1}\left(\sigma_{1}+\sigma_{2}\right) f_{2}\left(1-f_{3}\right)+a_{2} \sigma_{3} f_{1}\left(1-f_{2}\right)} \\
& \tau_{2} \cong \frac{a_{1}\left(\sigma_{1}+\sigma_{2}\right) f_{2}\left(1-f_{3}\right)+a_{2} \sigma_{3} f_{1}\left(1-f_{2}\right)}{a_{1} a_{2} \sigma_{2} \sigma_{3}} .
\end{aligned}
$$

Not much has been gained since the respective occupancies are still difficulty related to the rate constants. Almost all theories use the BA however. Applying this :

$$
\begin{aligned}
& \tau_{1} \cong \frac{f_{1} f_{2}}{a_{1}\left(n_{1}^{0}+n_{2}^{0}\right) f_{2}+a_{2} n_{3}^{0} f_{1}} \\
& \tau_{2} \cong \frac{a_{1}\left(n_{1}^{0}+n_{2}^{0}\right) f_{2}+a_{2} n_{3}^{0} f_{1}}{a_{1} a_{2} n_{2}^{0} n_{3}^{0}}
\end{aligned}
$$

where the superscript refers to the equilibrium values. For comparable rates and for levels not too distinct in position :

$$
a_{2} n_{3}^{0} f_{1} \gg a_{1}\left(n_{1}^{0}+n_{2}^{0}\right) f_{2}
$$

which yields

$$
\begin{aligned}
\tau_{1} & \cong \frac{f_{2}}{a_{2} n_{3}^{0}} \\
\tau_{2} & \cong \frac{f_{1}}{a_{1} n_{2}^{0}} .
\end{aligned}
$$

Using (34) plus the BA, the time constants can then be written as

$$
\tau_{1} \cong \frac{1}{b_{2} N_{3}}, \quad \tau_{2} \cong \frac{1}{b_{1} N_{2}} .
$$

Note their resemblance with the Shockley lifetimes of holes and electrons in extrinsic n-type and extrinsic p-type material respectively [33]. Only for (58)-(60) one gets a clear physical picture of the origins of the time constant distribution. The approximations leading to these results are not always realistic.

For the n-dimensional case (next section) we use the general assumption that the free carriers interact with the states in the oxide. Applying this in the three level case means that $f_{1}=f_{2}=f_{3}=f$ with $a_{1}=b_{1}$ and $a_{2}=b_{2}$ (see eq. (34)). Together with the BA, the relaxation matrix becomes :

$$
\mathbf{M}_{13}=\left[\begin{array}{ll}
a_{1}\left(N_{1}+N_{2}\right), & a_{1} N_{1} \\
-a_{2} N_{3}, & a_{2}\left(N_{2}+N_{3}\right)
\end{array}\right]
$$

and the time constants :

$$
\begin{aligned}
\tau_{1} & \cong \frac{1}{a_{1}\left(N_{1}+N_{2}\right)+a_{2}\left(N_{2}+N_{3}\right)} \\
\tau_{2} & =\frac{a_{1}\left(N_{1}+N_{2}\right)+a_{2}\left(N_{2}+N_{3}\right)}{a_{1} a_{2} N_{2}\left(N_{1}+N_{2}+N_{3}\right)} .
\end{aligned}
$$

For the rate constants that are not too different and applying the adiabatic assumption $\left(N_{3} \gg N_{1}, N_{2}\right)$ :

$$
\tau_{1} \cong \frac{1}{a_{2} N_{3}}, \quad \tau_{2} \cong \frac{1}{a_{1} N_{2}} .
$$

Under these conditions the spectrum can be written as (see eq. (45)) :

$$
S_{33}(f)=4 n_{1}^{0} \frac{\lambda_{1}}{\lambda_{1}^{2}+\omega^{2}}+4 n_{2}^{0} \frac{\lambda_{2}}{\lambda_{2}^{2}+\omega^{2}} .
$$

This is an interesting result. It shows under what conditions the superposition of spectra is allowed.

8. Discussion. - The mathematical analysis of one and two variable Markov processes (the $n$-variable case will be discussed in the following section) has been extensively dealt with on various occasions. The standard texts are those of [13] and [14]. The reason why we have reproduced certain results is threefold.

In the first place, we like to stress the simple form of the stationary variance in the multilevel case by using grand canonical variances in a consistent way. The intrinsic recombination variance, for instance, is identical with (18) if the BA is used and if the valence band is identified with the first level. Some explicit results have been obtained in [35] (appendix B). Again the BA is used however. The use of the BA may be the main reason why this symmetry in the variance never received much attention. This is probably due to the popularity of the quasi levels, since they imply the BA, thereby simplifying the analysis. If this symmetry is disregarded, it will be difficult to use the complicated expressions for a $1 / f$ theory. For the more involved cases their explicit calculation is usually avoided by evaluating the spectrum directly, using the relaxation matrix elements and those of the infinitesimal variances $\left(1 / 2 \mathbf{M}^{-1} \mathbf{B}\right.$ rather than $\left.\mathbf{C}\right)[13,14]$. As an example we take equation (16) of [29]. Electron-hole recombination with donors as a third level gave for the variance of the electrons :

$$
\overline{\Delta n^{2}}=\frac{1}{1-r^{2}}\left[\frac{1}{n_{0}}+\frac{1}{i_{0}}+\frac{1}{N-i_{0}}\right]^{-1}
$$




$$
\begin{array}{r}
r^{2}=\left[\frac{1}{i_{0}}+\frac{1}{N-i_{0}}\right]^{2}\left[\frac{1}{n_{0}}+\frac{1}{i_{0}}+\frac{1}{N-i_{0}}\right]^{-1} \times \\
\times\left[\frac{1}{p_{0}}+\frac{1}{i_{0}}+\frac{1}{N-i_{0}}\right]^{-1}
\end{array}
$$

where $n_{0}$ is the equilibrium value for the electrons and $i_{0}$ for the donors, with $p_{0}$ the hole equilibrium density. Identifying the electrons with the third level, the donors the second and the holes with the first one, this expression reduces to (our notation) :

$$
\overline{\Delta n_{3}^{2}}=\frac{n_{3}^{0}\left(n_{1}^{0}+\sigma_{2}\right)}{n_{1}^{0}+n_{3}^{0}+\sigma_{2}}
$$

As can be seen, the thermodynamic method from [29] implies the BA for the valence and the conduction band $\left(f_{1} \sim 1, f_{3} \ll 1\right)$.

In the second place, the complexity of the expressions for the time constants and/or variances makes it difficult to use the $1 / \tau$ idea effectively. Any simplification is thus welcome. Avoiding these analytical difficulties and assuming an overall relaxation is masking physical detail and leads. some times to a wrong interpretation. It is preferable to use the exact statistics (within a certain context of course, which is in our case a stationary and local Markov process) and to distinguish carefully between the various levels that one wants to take into account.

Thirdly, the treatments from $[13,14,29]$ and others make a free use of the Boltzmann Approximation. At the interface the situation is rather complex. If one accepts the idea of an amorphous transition region $[15,16]$ then the processes responsible for the excess noise suggest that the BA becomes inaccurate. The same holds for the states at the Fermi level interacting with the oxide states. Our treatment therefore avoids the BA, or equivalently, the use of quasi levels.

Although it is well known that a multi dimensional trapping process leads to a superposition of spectra, it has never been attempted to get an explicit result under certain circumstances. The problems are usually solved numerically. Surface related $1 / f$ theories make an extensive use of such an explicit superposition. It would be worthwhile to know under what conditions this occurs. The complexity of expressions like (42) is not very encouraging. It is therefore interesting to see that an adiabatic assumption already leads to equation (58).

9. Multi-level kinetics. - The literature of surface related $1 / f$ theories is not particularly generous with details as far as the origins of the socalled trapping effects are concerned. They are necessary for a statistical treatment. The modifications of the McWorther ideas ([5-10] a.o.) assume that the carriers tunnel directly form the conduction or valence band into socalled surface states located in the oxide. Their nature is not clear yet and their distance from the interface is thought to determine their capture and release rates. Other treatments introduce an intermediate state, often referred to as a fast interface state [10] or assume an effective use of the Fermi level [9]. On the other hand, these aspects require a different interpretation if one introduces the idea of a strong electron-phonon coupling near the interface [36, 37]. As was mentioned before, in this paper we are only interested in the statistical origins of the McWorther model assuming therefore a direct interaction in the usual sense with states in the oxide. Consider a n-type channel.

The following multilevel problem then emerges :

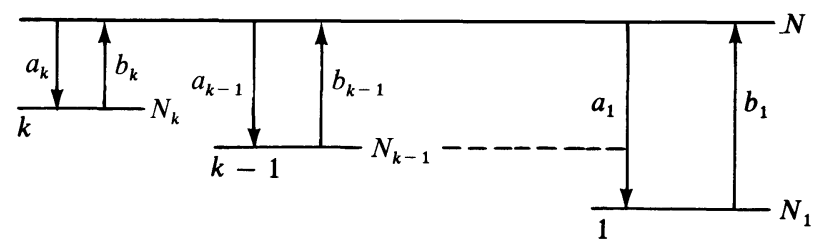

It is supposed that the levels do not interact with each other. For this $k$-level problem a particle constraint exists and there are $k-1$ time constants involved. In the limiting case the levels will be assumed to be closely spaced letting $k$ go to infinity. The rate equations governing the transitions are

$$
\begin{aligned}
& \frac{\mathrm{d} n_{1}}{\mathrm{~d} t}=a_{1} n\left(N_{1}-n_{1}\right)-b_{1} n_{1}(N-n) \\
& \vdots \\
& \frac{\mathrm{d} n_{k}}{\mathrm{~d} t}=a_{k} n\left(N_{k}-n_{k}\right)-b_{k} n_{k}(N-n) \\
& \frac{\mathrm{d} n}{\mathrm{~d} t}=\sum_{l=1}^{k} b_{l} n_{l}(N-n)-\sum_{l=1}^{k} a_{l} n\left(N_{l}-n_{l}\right) .
\end{aligned}
$$


Assuming a multi-dimensional O-U process, the perturbed quantities satisfy (eliminating the $k$ th level) :

$$
\begin{array}{cc}
\frac{\mathrm{d} \Delta n_{1}}{\mathrm{~d} t}=a_{1} N_{t} \Delta n-a_{1} N \Delta n_{1} \\
\vdots \\
\frac{\mathrm{d} \Delta n_{k-1}}{\mathrm{~d} t}=a_{k-1} N_{t} \Delta n-a_{k-1} N \Delta n_{k-1} \\
\frac{\mathrm{d} \Delta n}{\mathrm{~d} t}=-N_{t} \sum_{l=1}^{k} a_{l} \Delta n-a_{k} N \Delta n+\sum_{l=1}^{k-1}\left(a_{l}-a_{k}\right) N \Delta n_{l} .
\end{array}
$$

The nature of the McWorther type of treatments greatly simplify the $k$-dimensional problem, which is usually too complex to handle. For kinetics at the same energy the occupancies are the same for all levels. Using the equilibrium condition :

$$
\frac{a_{k}}{b_{k}}=\frac{n_{k}^{0}\left(N-n^{0}\right)}{n^{0}\left(N_{k}-n_{k}^{0}\right)}=\frac{f_{k}(1-f)}{f\left(1-f_{k}\right)}
$$

it follows that $a_{1}=b_{1}, a_{2}=b_{2}, \ldots a_{k}=b_{k}$. In deriving (70) the BA has been used together with a uniform distribution in the oxide $\left(N_{1}=N_{2}=\cdots=N_{k}=N_{t}\right)$. This is common practice in surface related theories. The relaxation matrix can now be written as

$$
\mathbf{M}=\left[\begin{array}{ccccc}
a_{1} N, & 0, & 0, & \ldots, & -a_{1} N_{t} \\
0, & a_{2} N, & 0, & \ldots, & -a_{2} N_{t} \\
\vdots & & & \\
\left(a_{k}-a_{1}\right) N, & \left(a_{k}-a_{2}\right) N, & \ldots \ldots, & \sum_{l=1}^{k} a_{k} N_{t}+a_{k} N
\end{array}\right] .
$$

For the covariance matrix, the same idea is used as in the previous section for the three level problem :

$$
\begin{aligned}
\overline{\Delta n_{1}^{2}} & =\frac{\sigma_{1}\left(\sigma+\sigma_{2}+\sigma_{3}+\cdots+\sigma_{k}\right)}{\sigma+\sigma_{1}+\sigma_{2}+\sigma_{3}+\cdots+\sigma_{k}} \\
\overline{\Delta n_{1} \Delta n} & =\frac{-\sigma \sigma_{1}}{\sigma+\sigma_{1}+\sigma_{2}+\sigma_{3}+\cdots+\sigma_{k}} .
\end{aligned}
$$

The adiabatic assumption converts (73) and (74) into :

$$
\overline{\Delta n_{1}^{2}} \cong \sigma_{1}, \quad \overline{\Delta n \Delta n_{1}} \cong-\sigma_{1}, \quad \overline{\Delta n_{1} \Delta n_{2}} \cong \frac{-\sigma_{1} \sigma_{2}}{\sigma} .
$$

For a uniform distribution of trapping centres in the oxide, the covariance matrix then becomes :

$$
\mathbf{C}=\left[\begin{array}{cccccc}
\sigma_{0}, & \frac{-\sigma_{0}^{2}}{\sigma}, & \frac{-\sigma_{0}^{2}}{\sigma}, & \frac{-\sigma_{0}^{2}}{\sigma}, & \ldots, & -\sigma_{0} \\
\frac{-\sigma_{0}^{2}}{\sigma}, & \sigma_{0}, & \frac{-\sigma_{0}^{2}}{\sigma}, & \frac{-\sigma_{0}^{2}}{\sigma}, & \ldots, & -\sigma_{0} \\
\frac{-\sigma_{0}^{2}}{\sigma}, & \frac{-\sigma_{0}^{2}}{\sigma}, & \sigma_{0}, & \frac{-\sigma_{0}^{2}}{\sigma}, & \ldots, & -\sigma_{0} \\
\vdots & & & & & \\
-\sigma_{0}, & -\sigma_{0}, & -\sigma_{0}, & \ldots \ldots \ldots . ., & k \sigma_{0}
\end{array}\right]
$$

since $\sigma_{1}=\sigma_{2}=\cdots=\sigma_{0}$. As can be seen from (29) the matrix $\mathbf{M}+j \omega \mathbf{I}$ needs to be inverted. Since we are 
interested in the spectral density of $n$, only the last row of this matrix will be considered. Denoting its elements by $a_{k 1}, a_{k 2}, \ldots a_{k k}$ respectively, the cofactors of $a_{1 k}, a_{2 k}, \ldots a_{k k}$ are needed. As an example, the cofactor of $a_{1 k}$ is :

$$
\begin{array}{||ccccc||}
0, & a_{2} N+j \omega, & 0, & \ldots, & 0 \\
0, & 0, & a_{3} N+j \omega, & \ldots, & 0 \\
0, & 0, & 0, & \ldots, & 0 \\
\vdots & \vdots & \vdots & & \vdots \\
0, & 0, & 0, & \ldots, & a_{k-1} N+j \omega \\
\left(a_{k}-a_{1}\right) N, & \left(a_{k}-a_{2}\right) N, & \left(a_{k}-a_{3}\right) N, & \ldots, & \left(a_{k}-a_{k-1}\right) N
\end{array} \| .
$$

The other cofactors can be found in a similar way. The total determinant is more involved :

$$
\begin{aligned}
\|\mathbf{M}+j \omega \mathbf{I}\|=\left(a_{1} N+j \omega\right) & \left(a_{2} N+j \omega\right) \ldots\left(a_{k-1} N+j \omega\right)\left[\sum_{l=1}^{k} a_{k} N_{t}+a_{k} N+j \omega\right]+ \\
& +\left(a_{1} N+j \omega\right)\left(a_{2} N+j \omega\right) \ldots\left(a_{k-2} N+j \omega\right) a_{k-1} N_{t}\left(a_{k}-a_{k-1}\right) N \\
& +\left(a_{1} N+j \omega\right)\left(a_{2} N+j \omega\right) \ldots\left(a_{k-3} N+j \omega\right)\left(a_{k-1} N+j \omega\right)\left(a_{k}-a_{k-2}\right) N a_{k-2} N_{t} \\
+\cdots & +\left(a_{2} N+j \omega\right)\left(a_{3} N+j \omega\right) \ldots\left(a_{k-1} N+j \omega\right)\left(a_{k}-a_{1}\right) N a_{1} N_{t} .
\end{aligned}
$$

It is difficult to work with this expression. Fortunately the adiabatic assumption makes $N \gg N_{t}$ sothat (78) simplifies to :

$$
\|\mathbf{M}+j \omega \mathbf{I}\| \cong\left(a_{1} N+j \omega\right)\left(a_{2} N+j \omega\right) \ldots\left(a_{k} N+j \omega\right) .
$$

The spectrum follows from (69), (76), (77) and (79) by considering the last row of $(\mathbf{M}+j \omega \mathbf{I})^{-1}$ and the last column of (76) :

$$
\begin{gathered}
S_{n n}(f)=4 \operatorname{Re} \sigma_{0}\left[\frac{\left(a_{k}-a_{1}\right) N}{\left(a_{1} N+j \omega\right)\left(a_{k} N+j \omega\right)}+\frac{\left(a_{k}-a_{2}\right) N}{\left(a_{2} N+j \omega\right)\left(a_{k} N+j \omega\right)}+\cdots\right. \\
\left.\cdots+\frac{\left(a_{k}-a_{k-1}\right) N}{\left(a_{k-1} N+j \omega\right)\left(a_{k} N+j \omega\right)}+\frac{k}{\left(a_{k} N+j \omega\right)}\right] .
\end{gathered}
$$

A partial fraction expansion yields for :

$$
\frac{\left(a_{k}-a_{l}\right) N}{\left(a_{k} N+j \omega\right)\left(a_{l} N+j \omega\right)}=\frac{1}{a_{l} N+j \omega}-\frac{1}{a_{k} N+j \omega} .
$$

This converts $(80)$ in to :

$$
S_{n n}(f)=4 \sigma_{0} \operatorname{Re}\left[\frac{1}{a_{1} N+j \omega}+\frac{1}{a_{2} N+j \omega}+\cdots+\frac{1}{a_{k} N+j \omega}\right] .
$$

For $k=2$ this result reduces to the one obtained in the three level case with $\lambda_{1}=a_{1} N$ and $\lambda_{2}=a_{2} N$, with an appropriate choice of the levels and applying the BA. Taking the real part :

$$
S_{n n}(f)=4 \sigma_{0} \sum_{l=1}^{k} \frac{a_{l} N}{\left(a_{l} N\right)^{2}+\omega^{2}} .
$$

Denoting $a_{l} N$ by $\lambda_{l}$, the covariance is obtained by reverting (83) :

$$
C_{n n}(t)=\sigma_{0} \sum_{l=1}^{k} \mathrm{e}^{-\lambda_{l} t}
$$

For $t=0$ we find the variance $k \sigma_{0}$ back again which also follows from (see eqs. (73) and (74)) :

$$
\overline{\Delta n^{2}}=\frac{\sigma\left(\sigma_{1}+\sigma_{2}+\sigma_{3}+\cdots+\sigma_{k}\right)}{\sigma+\sigma_{1}+\sigma_{2}+\cdots+\sigma_{k}}
$$

by using $\sigma_{1}=\sigma_{2}=\cdots=\sigma_{0}$ and the adiabatic assumption.

According to equation (83) it should now be possible to obtain a $1 / f$-type of spectrum by means of a suitable choice of the rate constants. In the theories of the McWorther type, the capture and release of the oxide states are supposed to vary exponentially with 
respect to their location. This is not the only way to obtain the required long time constants however. If we accept a strong electron phonon coupling at the surface together with the bond breaking idea from [36] than automatically a distribution of relaxation times arises. Also for this case an exponential dependency appears to be quite realistic.

For obtaining a $1 / f$ spectrum the rates $a_{l}$ cannot be incremented since this gives in the limit a white spectrum. In the McWorther context the traps are spatially uniformly distributed in the oxide sothat for this case the distance will be incremented in order to obtain in the limit an integral which can be solved. Writing :

$$
\tau_{l}=\left(a_{l} N\right)^{-1}=\tau_{0} \mathrm{e}^{\alpha y_{l}}, \quad 0<y_{l}<y
$$

converts (83) into :

$$
S_{n n}(f)=4 \sigma_{0} \sum_{l=1}^{k} \frac{\tau_{0} \mathrm{e}^{\alpha y_{l}}}{\omega^{2}\left(\tau_{0} \exp \alpha y_{l}\right)^{2}+1} .
$$

For surface carriers tunneling into the oxide states at a distance $y_{l}$, the tunneling constant is usually written as [38] (see also for further refs.) :

$$
\alpha=\frac{4 \pi}{\hbar}\left[2 m^{*} W\right]^{1 / 2} .
$$

The barrier $W$ is determined by the respective work functions and gaps of $\mathrm{S}_{\mathrm{i}} \mathrm{O}_{2}$ and $\mathrm{S}_{\mathrm{i}}$. In various calculations $\alpha \sim 2.10^{-8}$ and $y \sim 20 \AA$ sothat $\alpha y \sim 40$. Slicing up the total tunneling distance into $k$ sections, we write for $y_{l}=l \frac{y}{k}$ and convert (87) into :

$$
S_{n n}(f)=4 \sigma_{0} \frac{k}{y} \sum_{l=1}^{k} \frac{y}{k} \frac{\tau_{0} \exp \left(\alpha l \frac{y}{k}\right)}{\left[\tau_{0} \exp \left(\alpha l \frac{y}{k}\right)\right]^{2} \omega^{2}+1} .
$$

The summation can be written as (Riemann integral) :

$$
\begin{gathered}
\operatorname{Lim}_{k \rightarrow \infty} \sum_{l=1}^{k} \frac{y}{k} \frac{\tau_{0} \exp \left(\alpha l \frac{y}{k}\right)}{\left[\tau_{0} \exp \left(\alpha l \frac{y}{k}\right)\right]^{2} \omega^{2}+1}= \\
=\int_{0}^{y} \frac{\tau_{0} \mathrm{e}^{\alpha x}}{\left[\tau_{0} \mathrm{e}^{\alpha x}\right]^{2} \omega^{2}+1} \mathrm{~d} x .
\end{gathered}
$$

It is of course assumed that the states are sufficiently densely spaced in the oxide. For $\alpha y \sim 40$ the integral simply converts into $(4 \alpha f)^{-1}$. Since the BA applies, the prefactor $k \sigma_{0}$ represents the total number of occupied states in the oxide (denoted by $N_{\text {ox }}$ ) at a certain energy. The final result (83) can now be written as :

$$
S_{n n}(f)=\frac{N_{\mathrm{ox}}}{\alpha y} \frac{1}{f} .
$$

For the current spectral density (see eq. (2)) we write :

$$
S_{I}(f)=\frac{I^{2}}{\langle N\rangle} \beta \frac{1}{f}, \quad \beta=\frac{N_{\mathrm{o} x}}{\langle N\rangle \alpha y}
$$

where $\langle N\rangle$ is the total number of carriers in the sample. From (92) it can be seen that the surface state efficiency idea from the model in section two is not so unrealistic. It mightbe worthwhile to evaluate the numerical order of magnitude of $\beta$. It is often called Hooge's constant, when the current spectral density is written in the form of (92). Its value is of the order of $2 \times 10^{-3}$ for various materials and substances. For a modern M.O.S. device of, say, $10 \times 10 \mu$ on a moderately doped substrate and with an oxide thickness of about $1000 \AA$ the number of free carriers will be of the order of $10^{7}$ and the state density (C-V plot) around $10^{10} \mathrm{~cm}^{-2} \mathrm{eV}^{-1}$. For an energy interval of $1 k T$ and taking the dimensions into account $\beta$ is about $10^{-5}-10^{-6}$ which is not so unrealistic at strong inversion [26]. Assuming charged dangling bonds at the origins of the slow states [36, 37], the trapdensity will be larger since $\mathrm{C}-\mathrm{V}$ plots only detect the net charge.

The above remarks are not intended to present equation (83) as the key result of some $1 / f$ theory. The interface is too complex to allow such a straight forward characterisation.

10. Discussion. - Although surface explanations are rather specific, there are certain strong arguments however (both physical and statistical) that can be found back in almost every $1 / f$ theory.

Examples are the superposition idea and the distribution of time constants. Their ad-hocness prevents a microscopic clarification of the origins of $1 / f$ noise and their relationship with more fundamental properties of the system remains unclear. Statistical mechanics, however, does not leave much room for a $1 / f$ process. This is particularly true for the single time constant approximation : (i) An entropy expansion shows the effective use of the central limit theorem and the relevant variables are Gaussian (ii) Onsager's relations, second quantization and quantum mechanics in general (Heisenberg picture for instance) assume microscopic linearity (iii) Liouville's theorem implies ergodicity. Linear response theory and irreversible thermodynamics extensively use these notions. The theory of stochastic processes shows however that a linear, stationary and Gaussian process is automatically Markovian, and therefore never yields $1 / f$ noise (exponential relaxation). The only way, within the context of these ideas, to obtain a $1 / f$ type of spectrum is then to consider projections of processes or introducing non-linearities. In the latter case it should then be distinguished between non-linearly transformed linearity or linearly transformed non-linearity. It is interesting to note that even for highly nonlinear processes a stationary Gaussian 
Markov process is a quite attractive approximation in the thermodynamic limit and therefore not giving $1 / f$ noise. In this paper the projection idea is discussed. According to our final result, equation (83), it appears to be possible to obtain a $1 / f$-type of spectrum if our thermodynamic system (free density) interacts with numerous independent satellites. The obtained result resembles those from current surface theories. There are some distinct differences however.

The averaging in surface related theories (and others too) is done in two ways that are apparently different from one and another. Sometimes the fundamental spectrum is integrated over the oxide distance, sometimes it is assumed in an ad-hoc way that a $1 / \tau$ distribution exists. In both cases, to our knowledge, it has never been questioned whether the superposition is a valid procedure in the statistical sense. Some intrinsic difficulties arise. As can be seen from the previous sections, introducing extra levels complicates the analysis hopelessly if the problem is to be treated exactly. On the other hand, what exactly really means is not clear at all. Even if one could find the complete solution in the G-R sense, it is still an approximate way of reasoning since (i) The rates are supposed to be additive which may not hold in strongly interacting systems (ii) The non-linear statistics caused by the exclusion principle are linearized (iii) Sharply peaked transition probabilities are assumed which limits the treatment to Markov processes with local changes. It remains to be seen however, if all the imposed conditions are realistic at the interface. An integration over the oxide distance implies the validity of these assumptions.

Assuming an ad-hoc $1 / \tau$ distribution for the timeconstants suggests that the basic spectrum (representing an O-U process [11]) needs to be averaged, or in other words, the spectrum represents a random function (transformed random variable). Two difficulties arise with this picture. In the first place, the procedure requires independent processes. This is usually accounted for by stating that the trapping in one surface element is independent from another and that each element has its own time constant $[4,6,39]$. For a density of states which is low enough, these interactions are probably independent. This is not enough however. For the external leads only the effects of some sort of pooled output of these elementary area's is sensed. The question is therefore whether the independent trap centres can be added statistically since they are connected with each other by means of the free carriers (pooled output). This is certainly not the case without the BA or an Adiabatic Assumption (AA). Statistically speaking, on those occasions where it is averaged added or integrated the relaxation matrix (72) needs to be diagonally sharp. As can be seen from (72), this is the case when the AA applies and with non-interacting subsystems.

The second difficulty is of a thermodynamic kind. Averaging means that numerous processes are involved. In the single time constant approximation it was shown in [11] that the resulting process did not belong to the phenomenological theory of irreversible processes of Onsager-Machlup. The relevant variable (free or trapped carriers) then belongs to an open system and is a projection of some set of variables that need to be complete in order to satisfy the Onsager-Machlup (O-M) theory. That this can yield a $1 / f$ type of spectrum is shown by equation (83).

Although this superposition resembles the $1 / \tau$ idea, the latter is thermodynamically unrealistic. It implies a random spectrum (function of the random variable $\tau$ ) and therefore the variance of a variance (not to be confused with variance noise). Classically, a thermodynamic system in steady state or in equilibrium includes a time independent phase space density or density matrix, Fluctuation-Dissipation, Equipartition, Liouville space etc. and therefore excludes a fluctuating mean since the above notions require ergodicity of the system under study.

In our case, the treatment is Markovian and stationary, whereas G-R theory is fully compatible with the O-M theory. It. suggests that for a thermodynamic system that interacts with numerous small satellites, a natural superposition of spectra occurs with a two-level variance (eq. 18) that converges in the non-degenerate case (BA) to the total number in the satellites. The superposition yields a $1 / f$-type of spectrum if the relaxations depend exponentially upon some parameter. This can be the oxide distance, hopdistance, gap energy, barrier energy, diffusion length etc. The $1 / \tau$ probability density function idea does not exist. This is also satisfying since a normalising constant is physically a difficult quantity to explain. The final result arises from a process that is not in equilibrium however. In equilibrium the noise is determined by the velocity spectral density of the free carriers [14] which is classically not perturbed by the trapping kinetics.

These notions imply the following inconsistencies for the model of section two. The autocovariance of equation (1) suggests an O-U process. This is not in accord with the non-Gaussian variance of equation (3). The time constant of equation (4) implies a heatbath (linearization and the BA). This is not consistent with the assumption that the free density has as a variance equation (3). The averaging yields a nonMarkovian process that does not satisfy the O-M theory [11]. Extra complications arise in a real device with respect to the classical treatment for a strong electron-phonon coupling $[15,16]$. Note however that equations (6) and (7) are identical with equation (92) if $\alpha y=\ln \tau_{2} / \tau_{1}$ and $\beta(\bar{N})=N_{\mathrm{ox}} /\langle N\rangle$. The former identity is a formal one (spatial averaging $v$ s. ensemble average) whereas the latter could be interpreted as some surface efficiency.

Despite these limitations one may wonder why the $1 / \tau$ idea has been so successfull in the past. It even has been proposed as a more general explanation of 
the $1 / f$ phenomenon [20] and is still quite popular today $[18,19]$. It is suggested that this is caused by the implicit use of some strong arguments :

(i) The grand canonical variance is firmly rooted into the general theory of equilibrium statistical mechanics.

(ii) The $\mathrm{O}-\mathrm{U}$ process is some kind of central limit process for complicated statistical phenomena.

The various modifications of the McWorther model all use these points in one way or another. The main line of thought is a grand canonical variance multiplied by a mean spectrum. The decoupling of the variance and the relaxation is only valid however if the transition probabilities are sharp enough to diagonalise the relaxation matrix. This is possible within the G-R context, which underlies the conditions from section 3. Using these ideas, one obtains automatically the right order of magnitude for the $1 / f$ noise (See the numerical example following eq. (92)).

11. Conclusion. - Surface related $1 / f$ theories inherently use strong statistical arguments and impose interrelated conditions upon the fluctuating variable that are often difficult to maintain :

(i) A Heatbath Assumption is used.

(ii) The kinetic equations are linearized.

(iii) The Boltzmann Approximation is used for the heatbath.

(iv) Small deviations from equilibrium are assumed. (v) The variable is stationary, Gaussian but nonMarkovian.

(vi) The system is not in Equilibrium.

(vii) The microscopic processes are additive.

Further objections with respect to existing theories are :

(i) The variance is usually excluded from the averaging.

(ii) The ad-hoc $1 / \tau$ assumption is thermodynamically unrealistic.

(iii) The Heatbath Assumption and the Boltzmann Approximation are not always satisfied.

(iv) Confusion exists about the interaction with the interface (the choice of the time constant determines the statistics).

(v) The Physical picture contradicts sometimes the statistical one (Physically Gauss, statistically nonGauss for instance).

The method can be summed up as follows :

(i) Consider a sufficiently large but weakly interacting system (conditions for superposition are then satisfied).

(ii) Look for an exponential rate dependency and calculate the mean spectrum.

(iii) The final spectrum is proportional to the sum of the Grand Canonical variances of the systems in the Heatbath multiplied by the mean spectrum.

Heuristically this can be stated as follows : Number Spectral Density $=$ Total number trapped $*$ Mean Spectrum, since the grand canonical variance is poissonian for the BA.

\section{References}

[1] SuRdin, M. M., J. Phys. Radium 10 (1939) 188.

[2] VAN Der Ziel, A., Physica 16 (1950) 359.

[3] Du PrÉ, F. K., Phys. Rev. 78 (1950) 615.

[4] McWorther, A. L., In : Semiconductor Surface Physics, Ed. R. N. Kingston et al. (Univ. Penss. Press) 1957.

[5] Christensson, S. et al., Solid State Electron. 11 (1968) 796.

[6] Berz, F., Solid State Electron. 13 (1970) 631.

[7] Hsu, S. T., Solid State Electron. 13 (1970) 1451.

[8] Mansour, J. R. M. et al., Br. J. Appl. Phys. 2 (1969) 1063.

[9] VAN Der ZIEL, A., Solid State Electron. 17 (1974) 110.

[10] FUH, H. S., SAH, C. T., IEEE Trans. Electron. Dev., ED 19 (1972) 273.

[11] Walma, A. A., Revue Phys. Appl. 15 (1980) 1435.

[12] Onsager, L., Machlup, S., Phys. Rev. 91 (1953) 1505.

[13] VlIET, K. M. V., FASSET, J. R., In : Fluctuation Phenomena in Solids Ch. 7, Ed. R. E. Burgess (Ac. Press) 1965.

[14] LaX, M., Rev. Mod. Phys. 32 (1960) 25.

[15] Walma, A. A., ESSDERC, Munich, Germany (1979).

[16] Walma, A. A., Proc. 1/f Symposium p. 533, Orlando, USA (1980).

[17] Vliet, K. M. V., Proc. IRE 46 (1958) 1004.

[18] Dutra, P. et al., Phys. Rev. Lett. 43 (1979) 646.

[19] Celasco, M. et al., Phys. Rev. B 17 (1978) 2953.

[20] Van Der Ziel, A., 1/f Symposium, Tokyo, Japan (1977).

[21] Van Der Ziel, A., $1 / f$ Symposium, Orlando, USA (1980).

[22] Mott, N. F., Davis, E. A., Electronic Processes in NonCrystalline Materials (Clarendon Press) 1979.
[23] Scher, H., Montroll, E. W., Phys. Rev. B 12 (1975) 2455.

[24] Green, M. S., J. Chem 20 (1952) 1281.

[25] SAH, C. T., Proc. IEEE 52 (1964) 795.

[26] Vandamme, L. K. J., De KuYPer, A. H., 5th Int. Conf. on Noise in Phys. Systems, Bad-Nauheim, Germany (1978).

[27] Cox, D. R., Miller, H. D., The Theory of Stochastic Processes, (Chapman and Hall) 1965.

[28] MandL, F., Statistical Physics (John Wiley) 1973.

[29] Burgess, R. E., Proc. Phys. Soc. B 68 (1955) 66169 (1956) 1020.

[30] Wannier, G. M., Statistical Physics (John Wiley) 1966.

[31] Walma, A. A., 5th Int. Conf. on Noise in Phys. Syst., Bad Nauheim, Germany (1978).

[32] Bartlett, M. S., An Introduction to Stochastic Processes (Cambr. Un. Press) 1955.

[33] Shockley, W., Read, W. T., Phys. Rev. 87 (1952) 835

[34] SHOCKLEY, W., Electrons and Holes in Semiconductors (D. V. Nostrand) 1950.

[35] Lax, M., Mengert, P., J. Phys. Chem. Solids 14 (1960) 248.

[36] Mott, N. F., Adv. Phys. 26 (1977) 363.

[37] Walma, A. A., Proc. 1/f Symposium, p. 90, Orlando, USA (1980).

[38] Ronen, R. S., RCA Rev. 34 (1973) 280

[39] ZIEL, A. V. D., Noise : Sources, Characterization, Measurement (Prentice Hall) 1970.

[40] Papoulis, A., Probability, Random Variables and Stochastic Processes, (McGraw-Hill) 1965.

[41] Bartlett, M. S., J. R. Stat. Soc. B11 (1949) 211 